\title{
Library Based Modeling of Process Chains
}

\author{
G. Zülch and A. R. Fischer
}

ifab - Institute of Human and Industrial Engineering (Institut für Arbeitswissenschaft und Betriebsorganisation) University of Karlsruhe, D-76128 Karlsruhe, Kaiserstraße 12, Germany, phone: +49 721 608-4250, Email: gert.zuelch@mach.uni-karlsruhe.de

\begin{abstract}
This contribution gives an overview of the method Object-Oriented Modeling of Process Chains OMP. It is based on a library of process segments and allows an efficient configuration of complex process networks. The presented library represents a reference model, which is prepared to model the technical processing of customer oriented orders in a small series and one-of-a-kind production system.

After an introduction into the poblem an object oriented concept will be discussed. The important features of OMP will be described. Finally, the influence of the level of detail in modeling will be investigated with the help of simulation.
\end{abstract}

\section{Keywords}

Object-oriented modeling method, reference model for processes, system of predetermined times, modeling on different levels of detail, simulation.

\section{INTRODUCTION}

While on one hand, modeling means a simplification of the real system, on the other hand it is necessary to include all facts which are necessary to describe the dependencies of the system's entities. As a result, models of very complex systems are still complex themselves, especially if a good model quality is claimed. Thus, modeling means not only simplification and finding the main points of the system but as well requires the ability to handle the complexity of the model. In consequence it should be possible to choose any view on any level of detail which is included in a model representation. As a first step there is the task of creating a (detailed) model.

This paper focuses on the modeling of process networks, that represent the technical work-off of customers orders (see figure 1). The number of tasks in a process chain relates directly to the structure of the requested product, i.e. to the components and parts which have to be adapted to the special requirements. 


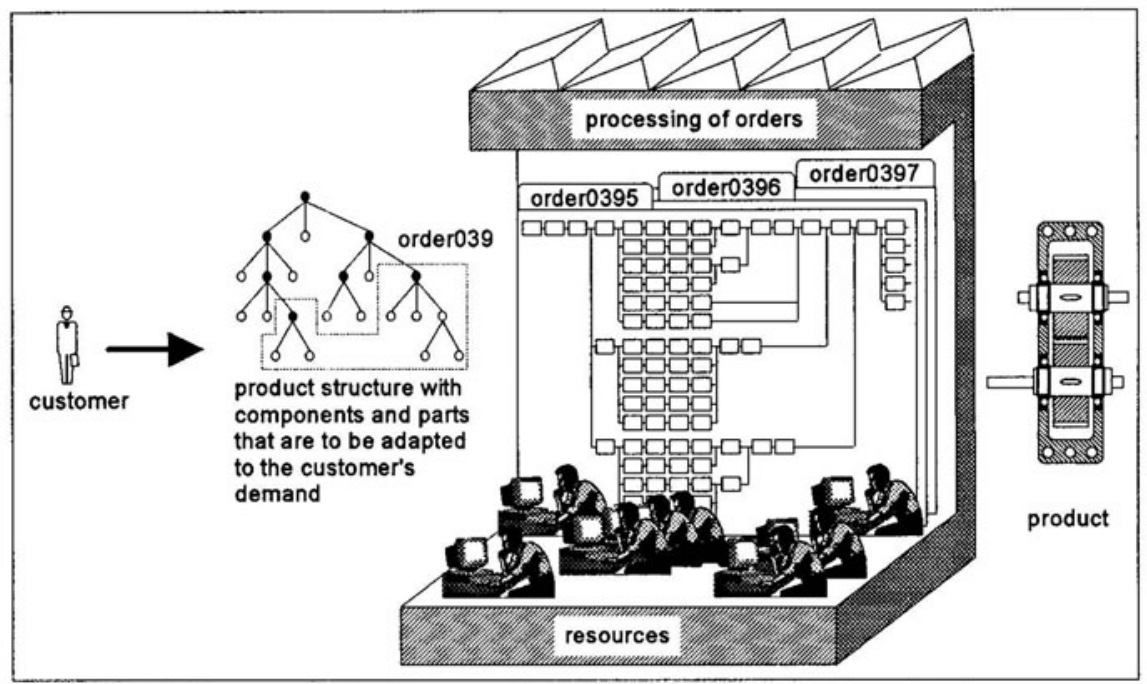

Figure 1 Order processing in a small series or one-of-a-kind production.

Besides creating the process network, i.e. finding all tasks that are necessary to fullfil the order and setting them in a logical relation to each other, it is necessary to guess the task's efforts, especially its operations durations. Both, configuration of the process network and the qualified guess of durations will be supported by the method Object-Oriented Modeling of Process Chains (OMP).

\section{OBJECT-ORIENTED MODELING OF PROCESS CHAINS (OMP)}

OMP is a general method to configurate complex processes based on a library. The latter includes a number of connected process segments and represents a reference model for processes. The concept of OMP is done under a strong objectoriented paradigm. Its main characteristics are abstraction, encapsulation, combination of data and behavior, inheritance and emphasis of object structure in contrast to procedure structure (Rumbaugh et al., 1991).

The objects of OMP are provided in figure 2. Its upper half includes all objects of the reference model, i.e. the objects for the process-library. The lower half of it includes all objects for the specific process-chains. They will be populated on basis of the reference processes. The symbol diagram (symbols following Rumbaugh, 1991 and Grobel, Kilger and Rude, 1992) shows all associations between the objects, e.g. the connection between process and its origin - the ref-process.

Modeling a specific order will be done by configuration of process-chains from the library. This task will be realized by the object's features. The procedure of modeling is specified by the complete specification of all objects, i.e. their attributes and their methods. While modeling, the objects call each other by their interfaces. As an example, the detail method of process is roughly described. 


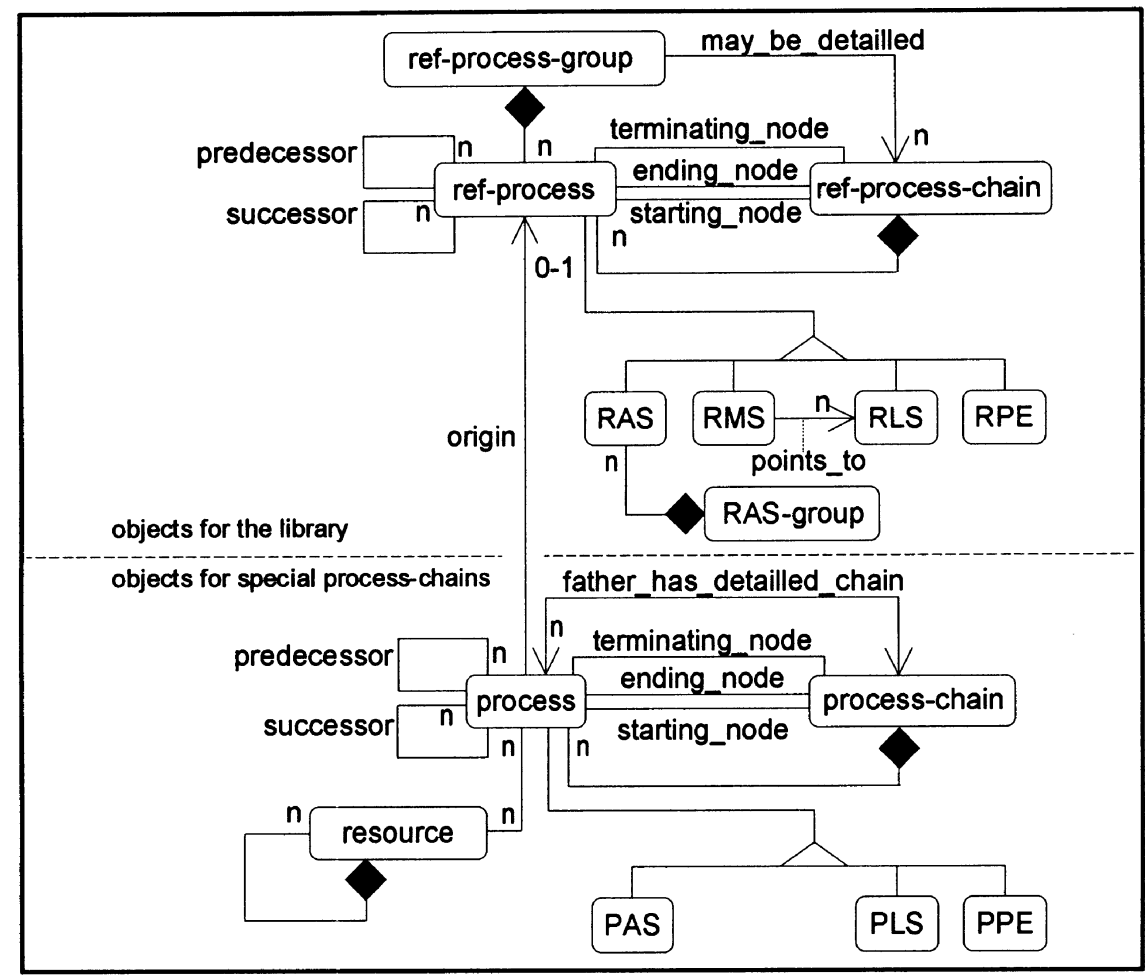

Legend:

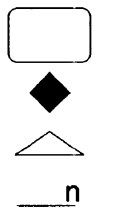

object (class)

aggregation (part_of)

generalization (inheritance)/specialization (is_a)

ref association with multiplicity (in respect to instances)

RAS reference-process_for_absolute_synchronization

RMS reference-mark_for_synchronization

RLS reference-process_for_local_synchronization

RPE reference-process_for_parallely_enlargement

PAS absolute synchronization-process

PLS local_synchronization-process

PPE parallely_enlargeable_process

Figure 2 Object classes in OMP.

A ref-process-chain and a time level (high, middel, low) are given as input. OMP provides possible chains, which could be choosen to detail the process: its origin 
ref-process belongs to a ref-process_group and this may be detailled by a couple of ref-process-chains (see figure 2). The information which chains are allowed to detail an actual process is represented in the library. Thus, the method works context sensitive.

\section{THE LIBRARY - A REFERENCE MODEL FOR ORDER PROCESSING}

As shown OMP is based on a library. Its population includes process segments and knowledge of their configuration possibilities. The reference model encloses process chains for the technical processing of customer oriented orders in a small series or one-of-a-kind production system for goods.

A process dictionary is worked out as an elementary basis. Its ref-processes belong to the 32 ref-process-chains of the library LIB. Some of them for example represent the procedure of designing a component $C$ or part $P$. Modeling a complex process-chain means specifying the customer variable part of a product structure. Table 1 shows an extract of the library. The grey marked ref-processes

Table 1 LIB-C: an extract of the library of process chains.

\begin{tabular}{lcccc}
\hline Processes in Process-chains & $H$ & $M$ & $L$ & ref-process-group \\
\hline LIB-C & & $\mathrm{R}$ & & \\
determining principle variant for C & 30 & 30 & 30 & \\
choosing solution principle for C & 90 & 60 & 30 & \\
determining DWS & 90 & 60 & 30 & \\
defining claims for adaptable sub- & 120 & 60 & 30 & \\
design-working spaces & & & & \\
defining interfaces for the SDW & 240 & 120 & 60 & \\
determining purchasing parts & 360 & 240 & 120 & \\
designing a component & $R$ & $R$ & $R$ & C-SOL, C-ALT, \\
& & & & C-REC-SOL; \\
& 1050 & 780 & 285 & P-REC-ALT; \\
designing a part & $R$ & $R$ & $R$ & DWS-ALT; \\
designing alternative DWS & 360 & 180 & 30 & \\
integrating moduls in DWS & 660 & 360 & 210 & CONF_CHECK; \\
checking conformity & 120 & 60 & 30 & \\
choosing alternative &.. &.. &.. & \\
... & & & & \\
\hline
\end{tabular}

Legend: ALT

DWS

$H, M, L$

$\mathbf{R}$

REC

SDW

SOL (design) alternative design-working-space plan time high, middle, low recursive structure is possible; plan time will be dependent from the component structure

Recursion sub-design-working-space (design) solution 
are detaillable - also by alternative chains. The latter ones are fixed by the refprocess-group. There exist as well ref-process-chains which can be detailled recursively (REC), because the process-chain structure occurs similar to the recursive product structure (a component includes again components). Basically, each process has three plan times. Thus, a synthetical determination of planning times goes along with the configuation procedure of a special order.

\section{MODELING ON DIFFERENT LEVELS OF DETAIL - INVESTIGATION WITH SIMULATION -}

With regards to the level of detail there are two aspects. The first one refers to the level of detail which should be modeled. The second one considers the level of detail which should be used for an application.

OMP allows a very efficient modeling, even if the level of detail is very high. Nevertheless, the developed library includes ref-process-chains for a rough modeling as well. It allows to stop the detailled configuration procedure at any level. Then, specifying the tasks for a component simply include functions instead of processes, e.g. rough designing, detail designing and operations planning. A function includes the tasks of all sub-components and parts which are included with the actual component. The single tasks, e.g. operations planning for part 4717 and for part 0814, can no longer be handled separately. Subsequently, the process orientation will be lost and replaced by a function oriented modeling. A parallel view of the tasks will become impossible as well as a realization of simultaneous engineering. An aggregated process only can be assigned to one organizational unit and an organizational distortion may occur. In addition, it becomes difficult to guess the plan time for a complex task or function.

As a first result it can be summarized that a high level of modeling brings advantages if there exists a method which allows an efficient modeling procedure. The idea is not to reduce the complexity but to give support to handle it with regards to modeling.

The second aspect refers to the application of a process-chain model. There exist a lot of topics. One could be to plan a suitable organizational structure (Zülch and Grobel, 1992; Grobel 1993). Then, the level of detail should be high, because the tasks should be assigned separately and under several aspects to the different organizational units. Only detailled process-chains enable to model a product or process oriented organization in contrast to a functional one.

Another application refers to resources coordination or order control. This means a dynamical processing or simulation of modeled process-chains. As an example an order with the adaption of 2 components is modeled. Each of them contains 1 sub-component and 2 respectively 3 parts. In addition also the design of alternatives is considered and modeled. To investigate the influence of the level of detail a model of the same thing, the customer order, was created on four different ways: (a) with OMP and use of the full specification of the product structure; (b) with OMP and selecting a more aggregated level of detail but with information of the complete specification; that is coming up from (a); (c) with 
OMP and the same level of detail as (b) but with chains for rough modeling and therefore, without full information; that is direct specification from the top to a certain specification of the product structure; (d) using (a) and aggregating its processes with respect to the given organizational structure; organization oriented modelling.

A good impression of the different modeling results is depicted in figure 3 . It shows how the aggregation of the processes reduces the possibilities of realizing a parallel processing. The models (b) and (c) in relation to (a) aggregate the product structure. The sub-components are no longer broken down. Here begins the transition to a function oriented modeling. For example, the operations plans of the first sub-component's 3 parts can no longer worked out separately. Case (d) is fully aggregated with respect to the organizational structure. The organizational structure consists of 9 departments and is the same for all four process-chain models. Function oriented modeling in this regard means modeling with respect to the given organizational structure. In figure 3, all tasks of stability calculation are done for all parts in a single department (see vertically framed processes). The processes just left to it in (b) and (c) can be done in two different departments: One creates operations plans for rotary parts the other one for cubic parts.

Aggregation can be done under several aspects, e.g. product structure oriented or with respect to a given organizational structure. For dynamical handling and simulation the latter one is useful and mostly necessary. Why that...?

... it is because of a probem with the full detailled process plan. It provides too much freedom for dynamical processing. A simulation program tries processing as parallel as possible (see figure 4). There is no restriction or dynamical rule to solve the problem. Defining rules would bring simulation more and more to a determined processing. This would be the end of simulation.

Processing an order means changing the view from the product structure to the resources. This makes it useful to build packages of the process-chain's tasks which can be made in direct sequence (or parallely) at one department. The problem is how to aggregate the tasks. The criterian may not only depend on the product structure. There is no hint how large a package of tasks should be. Thus, a method for useful aggregation remains as an open point.

The four ways of modeling lead to different system loads: (a), (b) and (d) have the same total load. They all are modeled with full information by the detailled structure from (a), i.e. a synthetic modeling with pre-determined durations is practiced.

An optical impression of the orderprocessing, done with the simulation program FEMOS (Grobel, 1992) is illustrated in figure 4. It is obvious that a higher level of detail goes along with more parallely processing.

A real and valid processing is positioned somewhere in between (a) and (d). The process chain from type (a) allows to process any degree of parallelity and sequency. The remaining problem is the dynamic rule how to control resources and orders. 
The level of detail may influence directly and massively absolute characteristics and even twice the goal accomplishments.

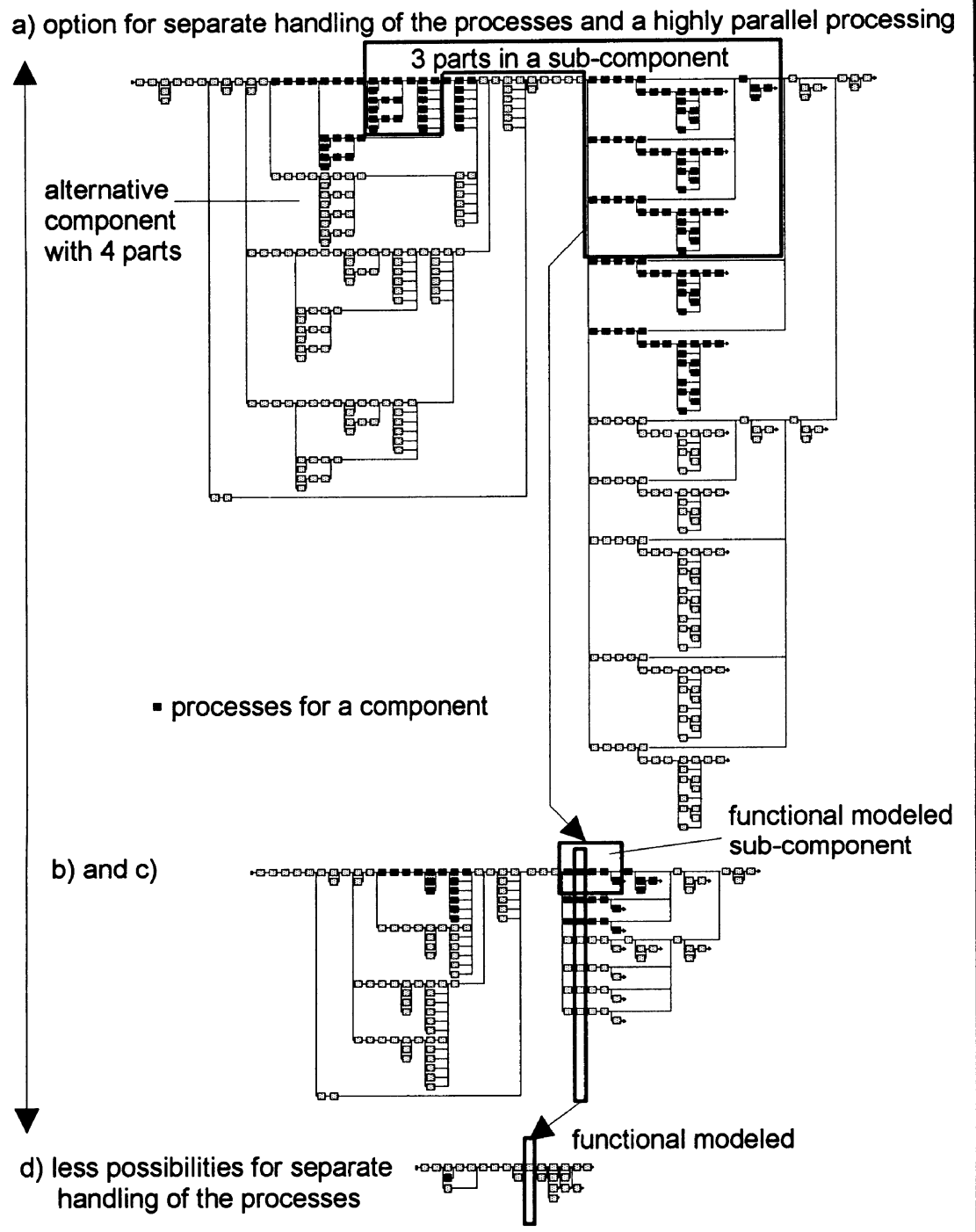

Figure 3 Modeled process chains for the same order 


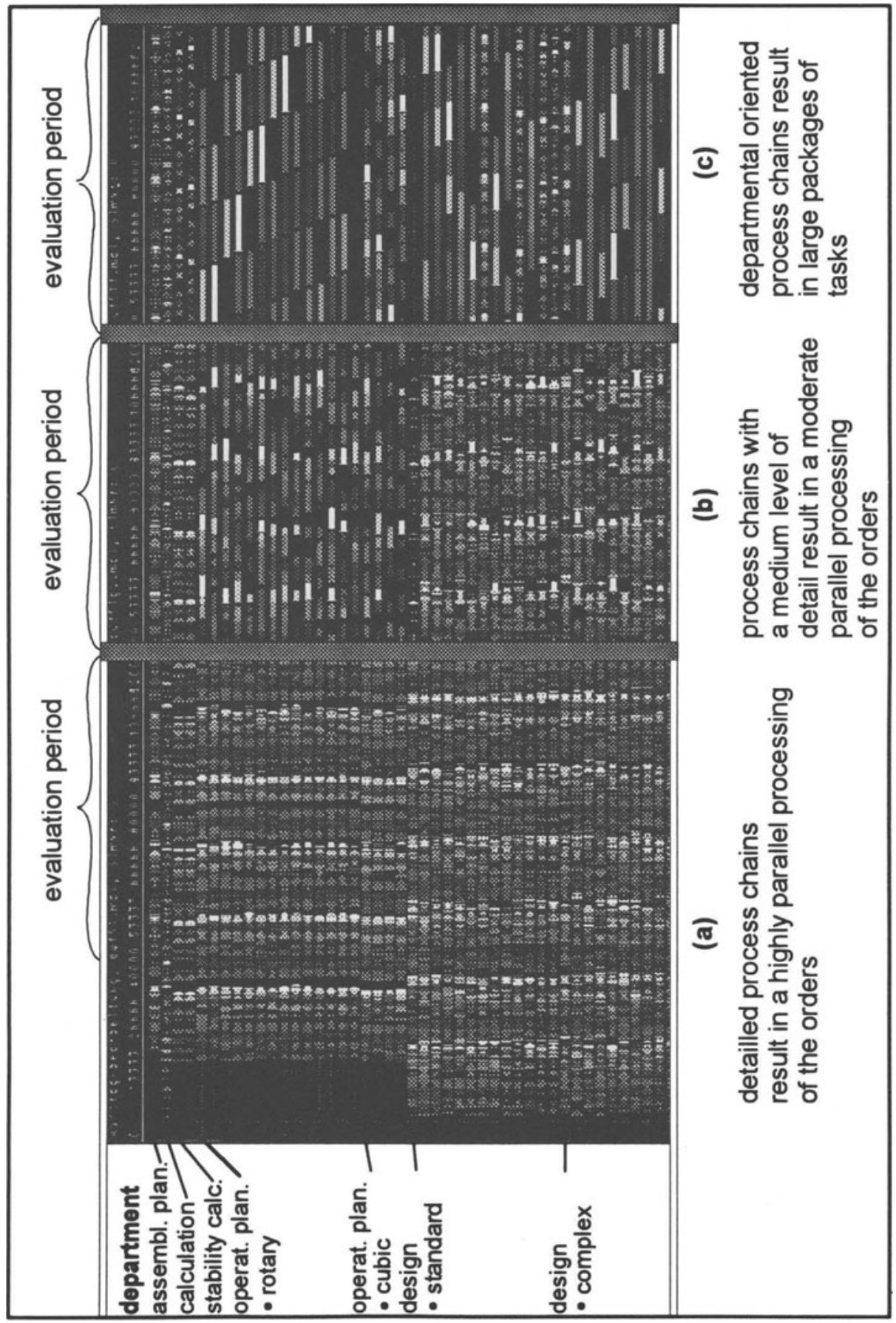




\section{SUMMARY AND OUTLOOK}

OMP allows a truely process or task oriented modeling of even complex processes. As the example has shown configurating a process e.g. for a component encloses rearly all tasks which are in connection with it. In contrast, function oriented modeling means an aggregation of tasks, mostly with respect to the orgnizational structure.

One of the major advantages of OMP is its context sensitive support for modelling. Thus, it enables a very efficient configuration procedure and provides a representation which includes several levels of detail with respect to the product structure. It supports as well a synthetic determination of plan times with predicted durations of tasks for higher aggregated processes.

The level of detail influences the modeling result and as well the dynamic order processing heavily. The rules for dynamic processing and simulation have to consider this influence. To assure predictability of system's behavior some more investigations are necessary. Simulation systems of today should be improved with respect to a valid dynamic processing of complex process chains.

\section{REFERENCES}

1. GROBEL, T. (1992). Simulation der Organisation rechnerintegrierter Produktionssysteme. Karlsruhe, Institut für Arbeitswissenschaft und Betriebsorganisation, Universität Karlsruhe, ISSN 0940 - 0559.

2. GROBEL, T; KILGER, C. and RUDE, S. (1992). Objektorientierte Modellierung der Produktionsorganisation, in Information als Produktionsfaktor (ed. W. Görke; H. Rininsland; M. Syrbe), Springer-Verlag, Berlin et al., 411419. (Informatik aktuell)

3. GROBEL, T. (1993). Analyse der Einflüsse auf die Aufbauorganisation von Produktionssystemen. Karlsruhe, Universität Diss., Institut für Arbeitswissenschaft und Betriebsorganisation, Universität Karlsruhe, ISSN 0940 0559 .

4. RUMBAUGH, J.; BLAHA, M.; PREMERLANI, W.; EDDY, F. and LORENSEN, W. (1991) Prentice Hall. Objectoriented modeling and design. Englewood Cliffs, NJ.

5. ZÜLCH, G. and GROBEL, T. (1992) Simulating the Departmental Organization for Production to Order, in 'One-of-a-Kind' Production: New Approaches (ed. B. E. Hirsch; K.-D. Thoben), IFIP Transactions B: Applications in Technology, B-2, Amsterdam et al: North-Holland, 177-193.

\section{BIOGRAPHY}

G. Zülch and A. R. Fischer, Institute of Human and Industrial Engineering (Institut für Arbeitswissenschaft und Betriebsorganisation - ifab), University of Karlsruhe, Germany. 
Prof. Dr.-Ing. Dipl.-Wirtsch.-Ing. G. Zülch, born in 1946, studied mechanical engineering at the University of Technology at Brunswich, and Industrial Engineering at the Superior School of Technology at Aix-la-Chapell, Germany. After 10 years of experience in research and industry, he became head of the then newly founded ifab-Institute at Karlruhe University in 1985.

Dipl.-Ing. A. R. Fischer, born in 1963, studied mechanical engineering (production technology) at the University of Karlsruhe, Germany, from 1983 to 1989. Since his graduation in 1989 he has been working as researcher at the ifab-Institute of Karlsruhe University. His research interests mainly concern modeling methods with respect to production systems and its analysis with support of simulation. 